\title{
Optical Material Characterisation of Prepreg CFRP for Improved Composite Inspection
}

\author{
Sebastian Meister ${ }^{1}$ (D) Jan Stüve ${ }^{1}$ (D) $\cdot$ Roger M. Groves $^{2}$ (D)
}

Received: 15 May 2021 / Accepted: 28 October 2021 / Published online: 24 November 2021

(c) The Author(s) 2021

\begin{abstract}
Automated fibre layup techniques are often applied for the production of complex structural components. In order to ensure a sufficient component quality, a subsequent visual inspection is necessary, especially in the aerospace industry. The use of automated optical inspection systems can reduce the inspection effort by up to $50 \%$. Laser line scan sensors, which capture the topology of the surface, are particularly advantageous for this purpose. These sensors project a laser beam at an angle onto the surface and detect its position via a camera. The optical properties of the observed surface potentially have a great influence on the quality of the recorded data. This is especially relevant for dark or highly scattering materials such as Carbon Fiber Reinforced Plastics (CFRP). For this reason, in this study we investigate the optical reflection and transmission properties of the commonly used Hexel HexPly 8552 IM7 prepreg CFRP in detail. Therefore, we utilise a Gonioreflectometer to investigate such optical characteristics of the material with respect to different fibre orientations, illumination directions and detection angles. In this way, specific scattering information of the material in the hemispherical space are recorded. The major novelty of this research are the findings about the scattering behaviour of the fibre composite material which can be used as a more precise input for the methods of image data quality assessment from our previous research and thus is particularly valuable for developers and users of camera based inspection systems for CFRP components.
\end{abstract}

Keywords Automated Fiber Placement · Inline inspection • Laser Line Scan Sensor • Optical material characterisation $\cdot$ Reflection $\cdot$ Transmission

\section{Introduction}

In advanced aviation and aerospace industry, lightweight composites are becoming more and more common. The Boeing 787 as well as the Airbus A350 XWB are typical aircraft types where the need for such composite components is rising [1,2]. Such structural parts

Sebastian Meister

sebastian.meister@dlr.de

1 Center for Lightweight Production Technology, German Aerospace Center, Ottenbecker Damm 12, Stade, Germany

2 Aerospace Non-Destructive Testing Laboratory, Delft University of Technology, Kluyverweg 1, 2629 HS Delft, The Netherlands 
are mostly manufactured using Carbon Fiber Reinforced Plastics (CFRP) owing to its outstanding stiffness and strength properties in comparison to metallic components. In order to reduce the costs of the complex fabrication procedure, efficient manufacturing techniques are of major interest. A suitable automated fibre laying technology is one way to increase production efficiency [3-5]. To comply with the high safety standards in the aerospace sector, a subsequent visual inspection is mandatory $[6,7]$.

These days, such manually conducted checks take from $32 \%$ [6] to 50\% [8] of the overall manufacturing time. In some cases, it is actually almost impossible to guarantee the necessary inspection accuracy as a result of the manual inspection procedure. Thus, the potential for improvement in quality and speed is enormous [8]. Due to their ability to record a three dimensional topological map of a surface, Laser Line Scan Sensor (LLSS) based inspection systems are particularly popular for automated inspection in fibre composite manufacturing $[4,9,10]$. These sensor systems project a laser beam at a specific incidence angle onto the fibre material surface. A camera senses the position of this laser line at a different viewing angle. However, the quality of the recorded data and thus the precision with which the laser line position is determined in the input image is potentially highly dependent on the material being viewed and its optical properties [11]. This influence is particularly relevant for very dark or strongly scattering surfaces such as CFRP.

To this end, some research has already carried out to investigate the reflectivity and emissivity of thermoplastic CFRP materials [12, 13]. However, these studies primarily examine the material behaviour in the thermal infrared spectrum. In addition, there is further research dealing with the heat transfer of thermoplastic composites when irradiated with a Xenon heating lamp $[14,15]$. Moreover, investigations on the reflection behaviour of thermoplastic materials during heating with lasers in the near-infrared spectrum are carried out [16].

However, only very little knowledge is available about the reflection behaviour of prepreg fibre materials in the visible wavelength range. Especially with the focus on inspection via a LLSS with a red semiconductor diode laser, detailed investigations have not been carried out so far. Such optical material properties are essential for the detailed modelling of an optical sensor system and the evaluation of the signal quality, as reported in our previous paper [17].

In this context, we perform detailed investigations of the optical material properties of Hexel HexPly 8552 IM7 CFRP thermoset prepreg material in this study. This is a commonly used publically available prepreg material in the aviation sector, which is why this material was chosen for the investigations in this paper. For this purpose, reflectance and transmittance measurements are performed for various fibre orientations, illumination angles and detection angles via a Gonioreflectometer. Building on this, our paper will answer the following research question: Which optical material characteristics of the Hexel HexPly 8552 IM7 prepreg Material are especially of interest for the automated LLSS based inspection of CFRP components?

The methodology in this study initially involves a summary of the state of the art. Then the procedure for measuring optical material properties with a Gonioreflectometer is described. The results are then discussed with respect to the application case of fibre composite inspection. As already mentioned in our paper Meister et al. [17] it requires considerable effort to configure an optical sensor and the respective evaluation algorithms for inspection in composite manufacturing. An appropriate sensor model and methods for assessing the signal quality, as described in our earlier paper [17], in combination with precise optical material properties as will be presented below, increases the precision of optical inspection in composite production and reduce the setup effort of an optical sensor. 
Accordingly, the findings from this paper add particular value for developers and users of LLSS based optical inspection systems in the automated production of CFRP components.

\section{Related Research}

This section summarises the state of the art in fibre placement and inspection technologies, as well as methods for measuring and modelling optical material properties. The outlined optical inspection techniques represent potential applications for which the knowledge of optical material properties as described in the second part of the state of the art section is very beneficial for increasing the measurement precision and reducing their setup efforts.

\subsection{Fibre Placement and Inspection Procedure}

In this section fibre placement and composite inspection procedures are outlined. These days different fibre placement technologies are available. The Automated Tape Laying (ATL) and Automated Fiber Placement (AFP) are the most frequently used processes of such a kind $[18,19]$. They deposit CFRP material in layers on a tool. The deposited composite consists of carbon unidirectional (UD) filaments. For prepreg composites these filaments are embedded in a polymer matrix [20]. A fibre layup process for the application of UD composite stripes is illustrated in Fig. 1. The AFP process is especially advantageous for the production of complex composite components. This technique is increasingly installed in industrial aerospace manufacturing [7]. In this respect we choose this AFP production use case to achieve a great transferability level of the findings from this study [21]. In this process an effector lays multiple narrow stripes of composite material (tows) along a preprogrammed course. During fibre deposition a separate component heats the fibre material to get improved tack properties [22]. Respectively, each structural component is made from several plies of prepreg material [20].

Due to the high safety requirements in aviation, a suitable inspection of each laid down ply is necessary. Hence, the inline inspection in the AFP manufacturing stage is highly discussed in industrial research. Therefore various sensor principles have been studied. The Fraunhofer Institute for Integrated Circuits (IIS) investigated polarisation camera sensors for this purpose [23, 24]. The Institute of Production Engineering and Machine Tools - University Hanover (IFW) and the National Aeronautics and Space Administration (NASA) examined thermographic camera monitoring devices [25-27]. Such monitoring principles acquire two dimensional inspection data.

Accordingly, InFactory Solutions [4], Profactor [28], Electroimpact [3, 5] and Danobat Composites [29] developed laser camera triangulation based inspection systems. Moreover, they applied such LLSS systems for inline inspection in AFP production. A key advantage

Fig. 1 AFP procedure for the application of UD prepreg tows. Therefore a device heats up the material and a compaction roller presses it onto the mould. In this Figure $\vec{v}$ denotes the layup velocity. $\vec{F}$ gives the compaction force

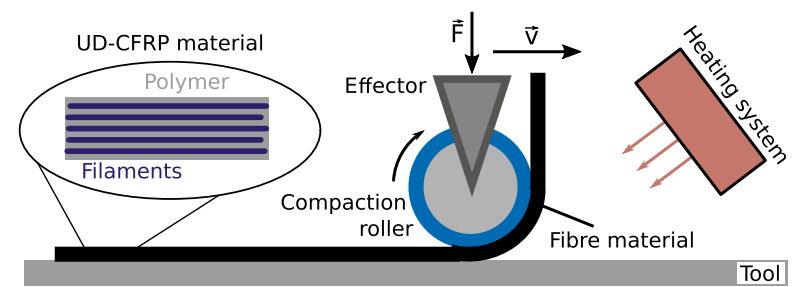


of such systems is the inherent determination of three dimensional surface information. For the AFP inspection this probably is the reason for success [4]. Additionally, Sun et al. [30] reviewed different monitoring devices for the fibre placement inspection.

For the LLSS inspection principle a laser line illuminates a surface and is reflected back to an imaging sensor. Sensor and Laser are aligned at an angle towards each other. To capture the three dimensional surface data the joined camera-laser device is moved in parallel to the surface, perpendicular to the laser line [9, 31].

In the following section, ways for determining optical material properties are presented.

\subsection{Principles for Measuring Optical Material Properties}

This section discusses the need for procedures to determine optical material properties. On this basis, techniques for the measurement of optical reflection and transmission properties are introduced. In this context, the measuring principle and different applications of a Gonioreflectometer are discussed.

Li et al. [32], Rabal et al. [33] as well as Martinez and Hartmann [34] all mentioned the need for tools to measure optical material properties especially for materials and coatings research. They also explain the relevance of spectrally resolved measured values for these optical material properties. Rabal et al. [33] measured the Bidirectional Reflectance Distribution Function (BRDF) in the spectral range from $380 \mathrm{~nm}$ to $780 \mathrm{~nm}$ with a spectral accuracy of $0.3 \mathrm{~nm}$. Martinez and Hartmann [34] also carried out BRDF measurements. Their utilised Gonioreflectometer system can perform spectrally resolved BRDF measurements in the wavelength range from $240 \mathrm{~nm}$ to $2000 \mathrm{~nm}$, with a resolution of 15 bit and a measurement uncertainty of $3 \mathrm{~nm}$. Li et al. [32] measure with their spectroradiometer in the spectral range from $380 \mathrm{~nm}$ to $760 \mathrm{~nm}$ with 1024 spectral bands. They digitise with 16 bit A/D resolution and have a spectral resolution between $0.82 \mathrm{~nm}$ and $3.3 \mathrm{~nm}$.

The subsequent section presents methods for modelling the material characteristics appropriately from the acquired optical measurement data.

\subsection{Modelling of Optical Material Characteristics}

In this section, characteristics of different surfaces and an appropriate modelling methodology for the corresponding optical properties are presented. In particular, bidirectional scattering and reflection functions are introduced. Furthermore, the propagation and interaction of a laser beam in CFRP material is discussed.

\subsubsection{Reflection, Scattering and Beam Propagation on Composite Material}

Initially, Forest et al. [35] briefly explained the reflection behaviour and modelling of specular, Lambertian and tranlucid surfaces. In contrast to this broad surface clustering, Schmitt et al. [31] provided basic concepts on scattering and beam propagation of laser light for CFRP material. They defined three different types for laser beam scattering at the filaments of a CFRP material. Additionally, Miesen et al. [36] examined the influence of measurement deviations from the scattering and beam propagation of a laser beam. They introduced appropriate error matrices in order to consider these influences in their model. Therefore they assumed that a laser beam propagates at a maximum twice the filament diameter inside the material before it is re-emitted from the surface. Otherwise the laser beam is absorbed from the material. As an extension to the studies from Schmitt et al. [31], Xu 
et al. [37] investigated the reflection behaviour of a single filament. Thus, they introduce a more detailed approach for modelling this reflection of a single fibre. Based on the work of Grouve [38], Stokes-Griffin and Compston [16] described qualitatively the reflective behaviour of UD CFRP materials. For this purpose, they first explained that the reflection characteristics of many individual fibres with the same fibre orientation accumulate in such a way that a single large cylindrical fibre can be investigated. This model is exemplary illustrated in Fig. 2 for the two extreme cases for a fibre orientation with $\gamma=0^{\circ}$ and for a fibre orientation $\gamma=90^{\circ}$. For the fibre orientation $\gamma=0^{\circ}$, the cylinder is illuminated obliquely in the fibre direction. Assuming we place a diffuse target behind the cylinder for which the normal of the diffuse target is parallel to the cylinder, then a semi-circular reflection of the incident light is imaged due to this illumination. However, assuming that the diffuse target and the incident beam direction remain constant, but the fibre cylinder is rotated to $\gamma=90^{\circ}$, the reflection of the incident light results in a straight vertical line on the diffuse target. Furthermore Riviere et al. [39] introduced the in-plane multispectral polarised BRDF modelling and measurement. They inverted experimental BRDF data with the aim to calculate the full BRDF and perform validation experiments on paint coatings. The principle of BRDF [40] is given by:

$$
f\left(\omega_{i r r}, \omega_{r e f}\right)=\frac{d L_{r e f}\left(\omega_{r e f}\right)}{E_{i r r}\left(\omega_{i r r}\right)}
$$

This equation consists of the reflected radiance $L_{r e f}$ and the incident irradiance $E_{i r r}$ for incident vector $\omega_{i r r}$ and reflection vector $\omega_{r e f}$. More detailed information on the analysis of reflected and scattered light measurements can be found in the book by Stover [41].

In order to model the scattering characteristics of a substrate they introduced a complex optical index which is composed of a real and an imaginary part. On this basis they specified the directional and diffuse components of a BRDF. Furthermore, Dingemans et al. [42] introduced a novel coefficient-independent scattering model to determine the thickness of a coating. They investigated the absorption and scattering behaviour of a

Fig. 2 The figure illustrates the imaging of the reflected irradiation from a cylinder on a diffuse target for both fibre orientations $\gamma=0^{\circ}$ and $\gamma=90^{\circ}$ with frontal lighting. This illustration is inspired by $[16,38]$
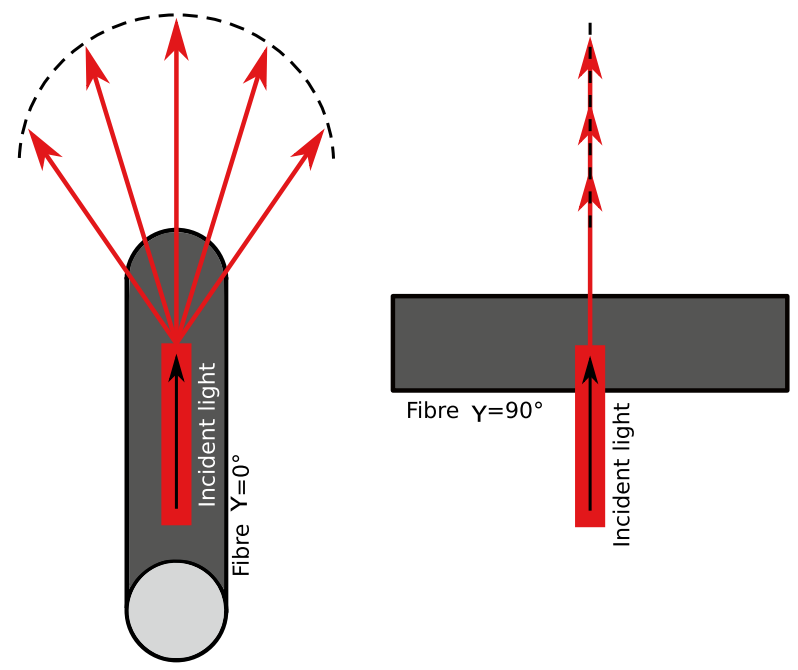
surface using their model. They utilised the Beer-Lambert law [43] to estimate the beam propagation along an optical path. Furthermore they applied the Kubelka-Munk model [44] to characterise light scattering within coatings and paint. With the aim to improve laser processing of CFRP, Hohmann et al. [45] applied and validated different methods for the simulation of the absorption, reflection and beam propagation characteristics of CFRP material. In their studies they investigated the beam propagation of a Gaussian laser beam inside a non-homogeneous material based on the Beer-Lambert law [43] with a one filament cylinder model. However, they discovered that this model for the beam propagation of a laser in CFRP has large errors and is therefore inappropriate for this application. Furthermore, they analysed several simulation techniques for calculating the diffraction parameters based on the scattering angle. Additionally, Zhang et al. [46] presented a method to simulate an image processing system for the inspection of a reflecting surface by means of ray tracing. The overall simulation consists of a defined camera model in combination with the reflection properties of the surface as well as the intensity and distribution of the reflected radiation as input parameters. A virtual reflecting surface with scratches was used for validation. In particular, they considered the Monte-Carlo ray tracing and used a pin-hole camera model. The BRDF was calculated with the aim to characterise the reflection properties of the surface considered.

Additionally, Stokes-Griffin and Compston [16] present in detail the interaction of a near infrared laser while heating CFRP material. In their study they researched the optical characteristics of a thermoplastic CFRP during laser illumination. In this respect they have also investigated certain absorption characteristics of the utilised composite.

In the following, a model for specifying the transmission of light in fibre composite materials is presented.

\subsubsection{Beam Transmission Model}

Iba and Kagawa [47] as well as Iba et al. [48] introduced the following formulation for modelling the light transmission $T$ in UD CFRP materials:

$$
T=\left[1-2 Q_{\text {ext }}\left(\rho_{r}\right)\left(\frac{\varphi_{c}}{\pi}\right)^{0.5}\right]^{\left(d_{m} / R_{f}\right)\left(\varphi_{c} / \pi\right)^{0.5}}
$$

In this equation, $Q_{\text {ext }}$ is the efficiency factor of the material. $\varphi_{c}$ represents the volume fraction of the composite. The parameter $d_{m}$ characterises the thickness of the sample. Moreover, $R_{f}$ gives the radius of a single fibre. Regarding Eq. 2 the challenge is to determine the efficiency factor $Q_{\text {ext }}$. The calculation of $Q_{\text {ext }}$ involves knowledge about the phase shift $\rho_{r}$ of the ray passing perpendicular to the aligned fibre through the filament. The parameter $\rho_{r}$ is calculated by:

$$
\rho_{r}=2 k R_{f}\left|n_{f}-n_{m}\right|
$$

Where the refractive index difference is $\Delta n=\left|n_{f}-n_{m}\right|$ and the individual refractive indices of the fibre is $n_{f}$ and the matrix material $n_{m}$. $k$ represents the wavenumber of the incident light.

For those reasons and due to the investigations of Pascual et al. [49] a very precise knowledge of $\Delta n$ is required. These values $\Delta n$ should be available with an accuracy of at least \pm 0.0001 . 


\subsubsection{Refraction, Diffraction and Interference}

In case a material is illuminated, the light is partly reflected and the other part is propagated inside the material. The ratio of reflected and transmitted radiation depends on the illumination angle. Thus, Snell's law is given by:

$$
n_{a} \sin (\beta)=n_{c} \sin \left(\theta_{c}\right)
$$

Here the relationship between incidence $\beta$ and refraction angles $\theta_{c}$ as well as the refraction indexes $n_{i}$ during the transition of a beam between two isotropic media is specified [16, 50]. Referring to Eq. 4, Eq. 5 gives the critical incident radiation for $\theta_{c}=90^{\circ}$. For irradiation at a larger angle of incidence, the radiation is fully reflected $[16,50]$.

$$
\beta_{R}=\arcsin \left(\frac{n_{c}}{n_{a}}\right)
$$

It should be noted that Snell's law is valid for isotropic media and thus can only be applied to CFRP in a limited manner.

Furthermore, the Fresnel equation specifies the reflection and transmission characteristics of electromagnetic radiation at the transition between two optical materials or fluids. For this purpose, Eq. 6 gives the relative reflectance $R_{s}, R_{p}$ for s- and p-polarised radiation.

$$
\begin{aligned}
R_{s} & =\left(\frac{n_{a} \cos (\beta)-n_{c} a}{n_{a} \cos (\beta)+n_{c} a}\right)^{2}, \\
R_{p} & =\left(\frac{n_{a} a-n_{c} \cos (\beta)}{n_{a} a+n_{c} \cos (\beta)}\right)^{2}, \text { with } \\
a & =\sqrt{1-\left(\frac{n_{a}}{n_{c}} \sin (\beta)\right)^{2}}
\end{aligned}
$$

The effective reflectance $R_{e}$ is calculated from the average of both values [50].

In the following section the experimental setup and the used methodology are outlined.

\section{Methodology}

This section explains the methodology for measuring the reflection and transmission properties of the considered composite material. Therefore, the material Hexply 8552 IM7 $[51,52]$ was measured at room temperature $20^{\circ} \mathrm{C}$. The Cured Ply Thickness (CPT) of this material is about $131 \mu \mathrm{m}$. The respective filament diameter is around $5.2 \mu \mathrm{m}$. This can also be assumed as the slit width between two filaments. Moreover, the material's volume fraction is $57.70 \%[51,52]$. However, the reflection behaviour is expected to vary for different material types and process temperatures. Nevertheless, the procedure for their measurement and modelling remains identical.

In order to ensure the transferability of the presented methodology for parameter estimation to other materials and processes, we utilised a Gonioreflectometer for our measurements. This device was introduced in Sect. 2.2. In particular we used an Opsira gonio'2pibsdf custom measuring instrument [53]. The corresponding assembly is shown in Fig. 3. This device consists of two individual spectroradiometers for the Visible Imaging System 
Fig. 3 The Goniometer gonio'2pi-bsdf setup is presented. It is shown the light source reflection mirror, the rotatable sample holder, and the two coupled spectrometers. $r_{i}$ are the individually rotatable axes

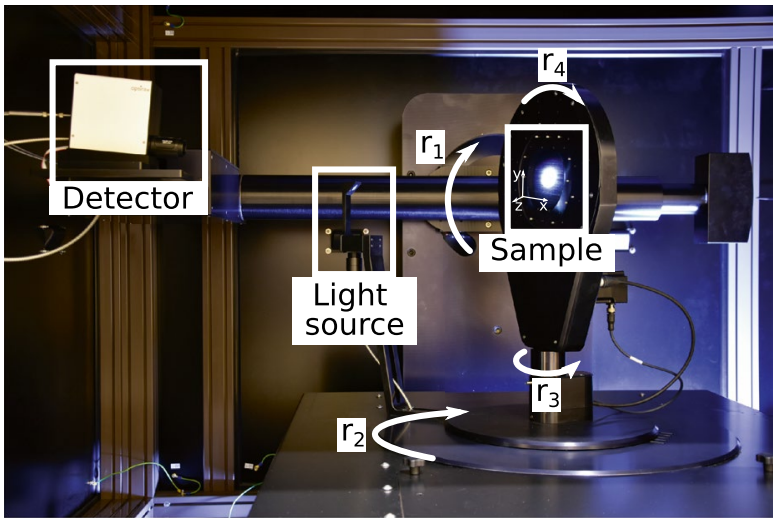

(VIS) and Near-Infrared (NIR) spectrum. Combined, both spectrometers cover the spectral range from $300 \mathrm{~nm}$ to $2400 \mathrm{~nm}$ with $1 \mathrm{~nm}$ spectral resolution. Each spectrometer performed a separate 16-bit A/D conversion. The angular resolution for the rotating axes is approximately $\mathrm{cd} / \mathrm{c}$. Thus, the combined rotation of all axes results in a spatial resolution of $\leq 0.03 \mathrm{~mm}$. The working distance of the spectrometer to the sample was about $650 \mathrm{~mm}$. The sample was illuminated with a $125 \mathrm{~W}$ Osram XBO 150 W/CR OFR Xenon lamp with a luminance of $20000 \mathrm{~cd} / \mathrm{cm}^{2}$ and an illumination spot diameter of around $25 \mathrm{~mm}$.

Under consideration of the output measurement data, an adjusted version of the BRDF from Eq. 1 in Sect. 2.3 was applied to characterise the material reflection properties. Keep in mind that for the investigations in this paper the use case of the laser illumination for an inspection system in the fibre placement process was considered. In this application, the viewing angle of the laser and the camera to the plumb line can only rotate in the $\mathrm{x}-\mathrm{z}$ plane. This plane is defined by the sample normal and the fibre orientation. Considering a line laser for measuring a surface topography, this laser line is rotated perpendicular to the fibre orientation. For this reason, at first, the reflection characteristics of the fibre material in the $\mathrm{x}-\mathrm{z}$ plane $\gamma=0^{\circ}$ were measured at various illumination angles $\beta$ and detection angles $\alpha$. Keep in mind, that for the considered use case, the applied lens of the LLSS has a certain field of view. Thus the pixels at the border of the measurement image result from a different laser beam angle to the optical axis than the measurement points located directly at the image centre. In order to investigate variations in the optical properties transverse to the fibre direction, the previous measurements are additionally carried out for the fibre orientation $\gamma=90^{\circ}$. In addition, transmission measurements were performed in order to investigate the refraction and the beam propagation inside the CFRP sample. For this purpose $\beta=$ const. $=180^{\circ}$ was set and $\alpha$ was rotated accordingly. For the reasons mentioned above, these measurements were carried out again for $\gamma=0^{\circ}$ and $\gamma=90^{\circ}$. The corresponding geometrical relations are illustrated in the Fig. 4. The aim was to determine the degree of reflectance of the material $\varrho_{m}$ from Eq. 7 for different illumination, detection and fibre orientation angles for the wavelength of the considered red semiconductor diode laser. In order to reach a higher precision of the measurements and to reduce disturbing influences, the measurement was performed integrally in the spectral range $\lambda=[640,660] \mathrm{nm}$. With the aim to determine the relative reflectance $\varrho_{m}$, the irradiance reflected by the material $E_{r m, \text { Отв }}$ was normalised 
Fig. 4 The schematic measurement setup is shown. $\alpha$ represents the angle between detector and plumb line. $\beta$ is the incident angle between illumination axes and plumb line. The angle $\gamma$ illustrates the rotation around the sample normal. This represents the fibre orientation. The angular rotation of $\alpha$ and $\beta$ is performed only in the $\mathrm{x}-\mathrm{z}$ plane

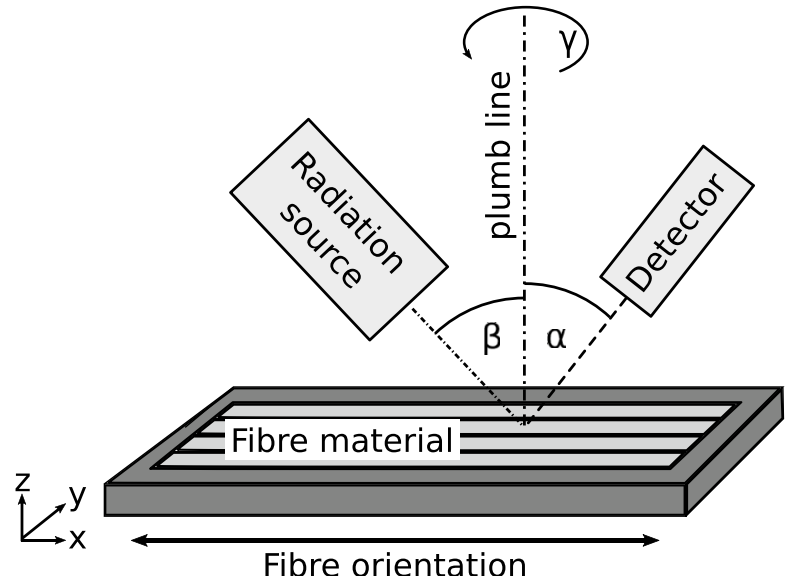

Fibre orientation

with the irradiance emitted from the light source $E_{l i \text { OTB }}$ over the given spectral range. Equation 7 presents this relationship as a kind of modified BRDF. The transmission was examined separately in order to investigate the beam propagation characteristics of the material.

$$
\begin{aligned}
& \varrho_{m}\left(\lambda=\text { const. } \alpha, \beta, \gamma=\left\{0^{\circ}, 90^{\circ}\right\}\right) \\
& =\frac{E_{r m, \mathrm{OTB}}(\lambda, \alpha, \beta, \gamma)}{E_{l i, \mathrm{OTB}}(\lambda, \alpha, \beta, \gamma)}
\end{aligned}
$$

For the reflection measurements in this paper, a $\alpha$ value range of $\left[0^{\circ}, 80^{\circ}\right]$ and for $\beta$ an interval of $\left[0^{\circ}, 70^{\circ}\right]$ was chosen. The detection angle $\alpha$ was rotated with a fine resolution of $1^{\circ}$. To increase the measuring speed and making use of the material symmetry, $\beta$ was rotated in steps of $5^{\circ}$. For measuring the transmission properties the incident angle remained constant at $\beta=180^{\circ}$. The angles $\alpha$ and $\gamma$ were rotated as previously explained. In the following section, the respective measurement results are presented.

\section{Results}

This section presents the reflection and transmission properties from the Goniometer measures previously outlined in Sect. 3. Figure 5 summarises the results for the reflection parameter $\varrho_{m}$ by considering different angles for $\alpha$ and $\beta$. The measurements are carried out for the sample rotations $\gamma=0^{\circ}$ presented in Fig. $5 \mathrm{~b}$ and $\gamma=90^{\circ}$ displayed in Fig. 5d. For a clearer overview, only the detection results in steps of $5^{\circ}$ are displayed. The measurement results for a fine detection resolution of $1^{\circ}$ are given in Fig. 5a for $\gamma=0^{\circ}$ and in Fig. 5c for $\gamma=90^{\circ}$. For these Figures, the detection angle $\alpha$ is displayed on the abscissa and the illumination angle $\beta$ on the ordinate. The light emitted on the material is partially reflected, transmitted, absorbed or propagated from the composite. Logically, the largest reflection values are achieved for the measurement of the specular reflection at equal detection and illumination angles $\alpha=\beta$. Due to the configured Goniometer measurement resolution of $1^{\circ}$, optical effects such as scattering can possibly lead to clipping of the actual peak value. 


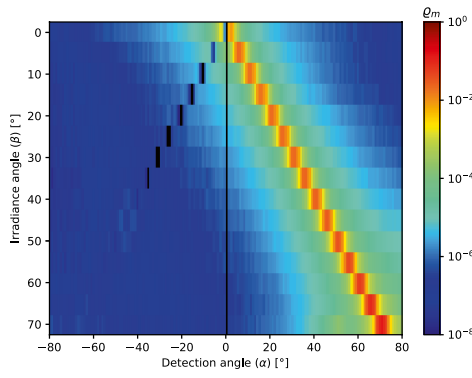

(a) Logarithm plot for the degree of reflection for the fibre ori-

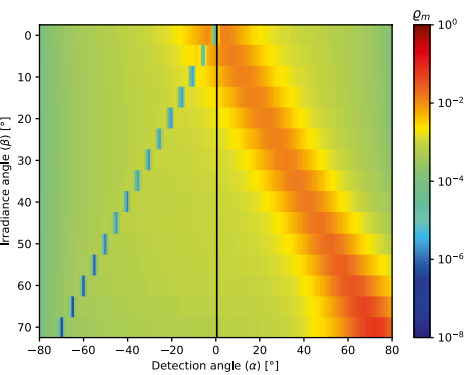

(c) Logarithm plot for the degree of reflection for the fibre ori (c) Logarithm pet

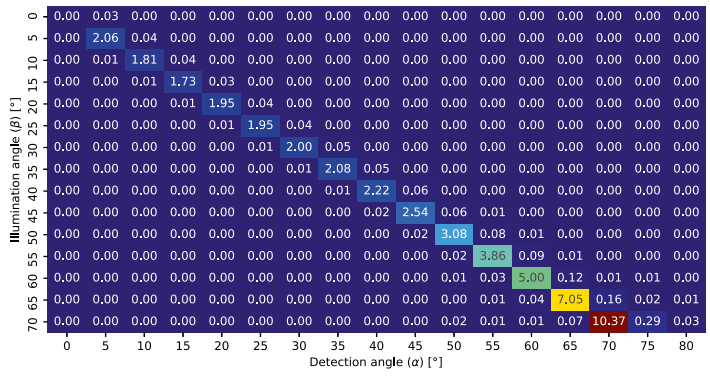

(b)

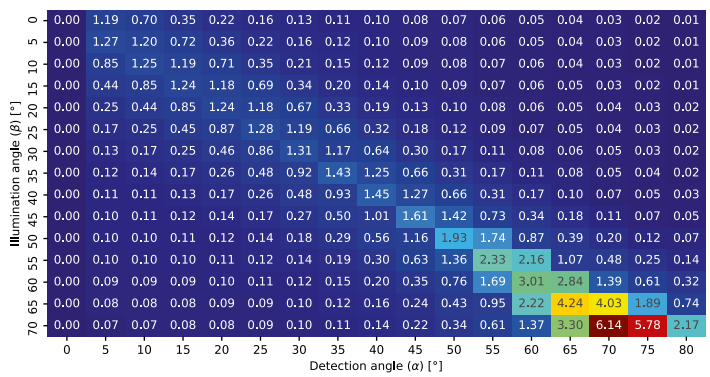

(d) Confusi

Fig. 5 The figure displays the reflection properties of the fibre composite material Hexcel HexPly 8552 IM7 for the fibre orientations $\gamma=0^{\circ}$ and $\gamma=90^{\circ}$. On the left side the degree of reflection with a detection angle resolution of $1^{\circ}$ and an illumination angle resolution of $5^{\circ}$ are shown. On the right side the corresponding confusion matrices are presented. The remaining incident light is either absorbed by or propagates inside the material. As explained in Sect. 2.3, the accumulated reflection of equally aligned fibres can be considered as the reflection from a large single cylinder. This yields a highly different variance in the degree of reflection over the detection angle $\alpha$, depending on the fibre orientation

However, the integral measurement over the relatively large light spot significantly reduces these local effects. Accordingly, we assume here that the maximum values measured at $\alpha=\beta$ are very close to the real maxima. Thus, clipping effects can be neglected. Shown in Fig. 5a these results vary from $1.73 \%$ to $10.73 \%$ relative reflection for $\gamma=0^{\circ}$. For $\gamma=90^{\circ}$ we see that the diagonal values range from $1.24 \%$ up to $6.14 \%$, which is presented in Fig. 5c. As previously mentioned, these reflection values are determined for the wavelength range $\lambda=[640,660] \mathrm{nm}$. However, it is noteworthy that for both fibre orientations on the diagonal $\alpha=\beta$ up to $40^{\circ}$ the $\varrho_{m}$ are quite constant. For a fibre orientation of $0^{\circ}$ these values range from $1.73 \%$ to $2.22 \%$. For $\gamma=90^{\circ}$ these values vary between $1.24 \%$ and $1.45 \%$. For $\alpha=\beta>40^{\circ}$ the diagonal values increase significantly faster up to the previously given maxima. Furthermore conspicuous for $\gamma=0^{\circ}$ in Fig. 5a is, that at an angle difference of more than $5^{\circ}$ which means $|\alpha-\beta| \geq 5^{\circ}$ almost no back reflection is detectable. For $\gamma=90^{\circ}$ this variance of the values is much greater. Depending on the angle, up to $|\alpha-\beta| \approx 15^{\circ}$ sensible measurement values can still be determined. With the aim of investigating this reduction of the reflection value, $\varrho_{m}$ is plotted along the illumination angles in the interval $\beta=[10,70]^{\circ}$ with step size $10^{\circ}$. The corresponding plots for $\gamma=0^{\circ}$ are shown in Fig. 6a. The graphs for $\gamma=90^{\circ}$ are displayed in the Fig. 6b. The full width at half maximum (FWHM) was taken as a reference value. This is the position at which the reflection value $\varrho_{m}$ has dropped by half. For the fibre orientation $\gamma=0^{\circ}$ we notice the FWHM $=2^{\circ}$ for all 
selected irradiation angles $\beta$. Assuming a Gaussian like intensity drop the standard deviation is $\sigma \approx 0.85^{\circ}$. This is clearly different for $\gamma=90^{\circ}$. Therefore the FWHM varies between $12^{\circ}$ and $16^{\circ}$. Therefore such a Gaussian like decrease of intensity results in a standard deviation interval of $\sigma \approx[5.10,6.79]^{\circ}$. However, it should be noted that the detection resolution of the angle $\alpha$ is only $1^{\circ}$ in this experiments. This can lead to smaller deviations of the FWHM. The individual plots clearly illustrate the reflectance behaviour of a cylinder described in Sect. 2.3 and displayed in Fig. 2. Keep in mind that for the measurements presented in Fig. 6, the detection and illumination angles for $\gamma=0^{\circ}$ are rotated in the direction of the fibre. For $\gamma=90^{\circ}$, both angles are rotated perpendicular to the fibre orientation. Thus, the shape of the curves presented here for a given $\gamma$ differs from the representation in Fig. 2 by $90^{\circ}$. Accordingly, for $\gamma=0^{\circ}$ a very narrow curve results and for $\gamma=90^{\circ}$ a significantly wider one. The FWHM values indicate this behaviour clearly.

In the two diagrams in Fig. 6, curve fittings of the individual maxima are carried out in accordance with the Fesnel Eq. 6 introduced in Sect. 2.3. For this purpose, we assumed that the surrounding air in the measurement setup has a refractive index of $n_{a}=1$. Thus, the refractive index of the composite $n_{c}$ is determined via the curve fitting, which aligns the Fresnel plot most precisely to the maxima. These values are presented in the legends of Fig. 6. We see that the refraction index varies for different fibre orientations, as in this case a non-isotropic material is measured. Accordingly, for the fibre orientation $\gamma=0^{\circ}$ the refraction index is $n_{c, 0}=1.3$. For a fibre orientation of $\gamma=90^{\circ}$ a refraction index of $n_{c, 90}=1.52$ is yielded. Moreover, on the basis of the modified Snell's law from Eq. 5 we can estimate the critical incident angle for total internal reflection $\beta_{R}$. Since Snell's law is only valid

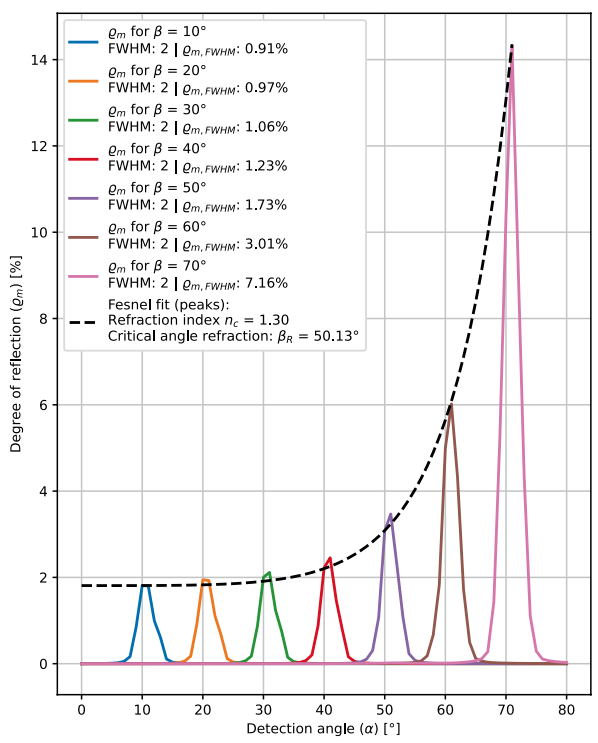

(a) Individual plots of the degree of reflection for equal illumination and detection angles $\alpha=\beta$ between $10^{\circ}$ and $70^{\circ}$ with a step size of $10^{\circ}$. The values are presented for the fibre orientation $\gamma=0^{\circ}$ and are given in $\%$.

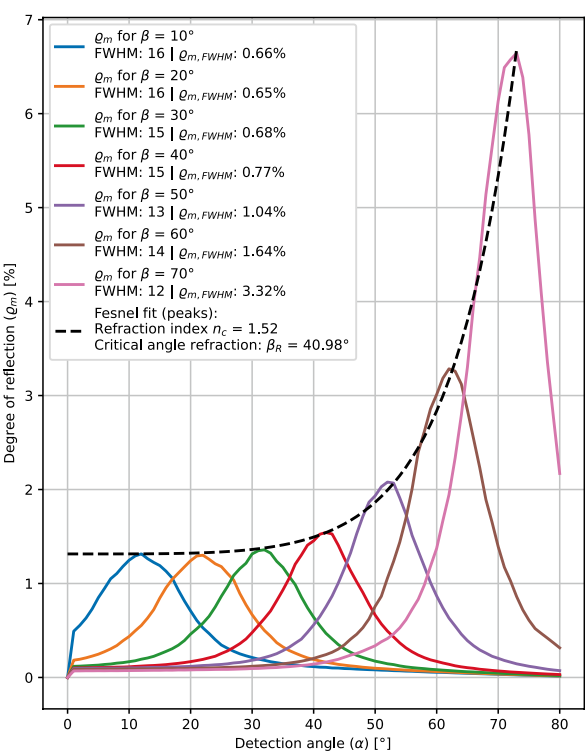

(b) Individual plots of the degree of reflection for equal illumination and detection angles $\alpha=\beta$ between $10^{\circ}$ and $70^{\circ}$ with a step size of $10^{\circ}$. The values are presented for the fibre orientation $\gamma=90^{\circ}$ and are given in $\%$.

Fig. 6 Plots of the reflectivity for equal incident and detection angles in the interval $\beta=[10,70]^{\circ}$ with a step size of $10^{\circ}$. The corresponding Fresnel fittings of the maximum values are visualised 
Fig. 7 Degree of transmission of the given composite material Hexcel HexPly 8552 IM7 for the fibre orientation $\gamma=0^{\circ}$ and $\gamma=90^{\circ}$ for a constant illumination angle of $\beta=180^{\circ}$. The maximum transmission values $T_{\max }$ and the corresponding detection angles $\alpha$ are given in the upper left corner of the figure. The measuring angle $\alpha$ -resolution is $0.25^{\circ}$. The values are given in $\%$

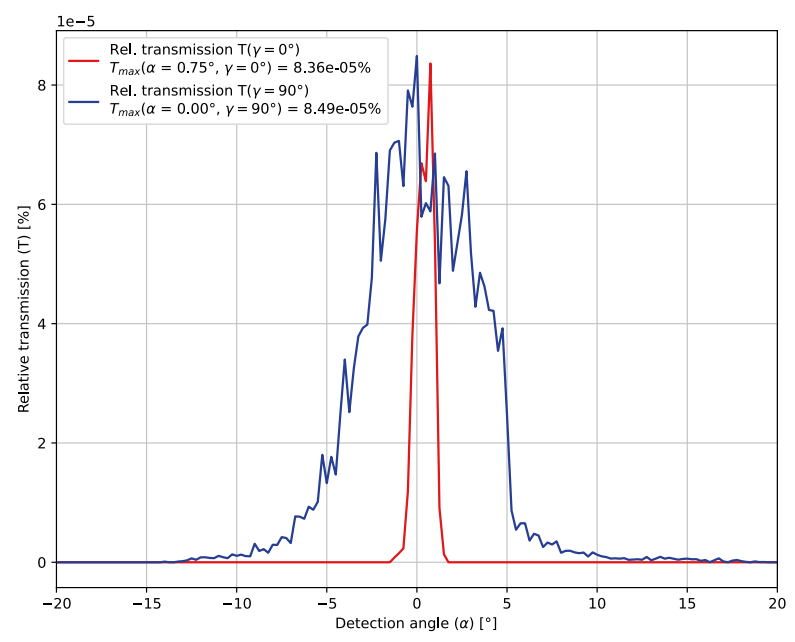

for isotropic materials, this is only a rough approximation for gaining understanding of the strong curve rise in the Fresnel curve fitting. For the fibre orientation $\gamma=0^{\circ}$ this gives $\beta_{R}=50.13^{\circ}$ and for $\gamma=90^{\circ}$ a $\beta_{R}=40.98^{\circ}$ is yielded. Additionally, the transmission of the material sample with a CPT of $131 \mu \mathrm{m}=1.31 \cdot 10^{-4} \mathrm{~m}$ are measured for both fibre orientations $\gamma=0^{\circ}$ and $\gamma=90^{\circ}$. The corresponding results are presented in the Fig. 7. The detection angles $\alpha$ are displayed on the abscissa. The relative transmission $\mathrm{T}$ is given on the ordinate. For a sample rotation of $\gamma=0^{\circ}$ transmission values $T\left(\alpha, \beta=180^{\circ}, \gamma=0^{\circ}\right)>0$ are measurable for a limited range of $\alpha \approx[-1.5,1.75]^{\circ}$. Moreover, considering a fibre orientation of $\gamma=90^{\circ}$ transmission values $T\left(\alpha, \beta=180^{\circ}, \gamma=90^{\circ}\right)>0$ are available in the much larger interval $\alpha \approx[-14.25,19.5]^{\circ}$. The maximum of the measured values for $\gamma=0^{\circ}$ is located at $\alpha=0.75^{\circ}$ with $T_{\max }\left(\alpha=0.75^{\circ}, \beta=180^{\circ}, \gamma=0^{\circ}\right)=8.36 \cdot 10^{-5} \%$. For $\gamma=90^{\circ}$ this maximum is at $\alpha=0^{\circ}$ with $T_{\max }\left(\alpha=0^{\circ}, \beta=180^{\circ}, \gamma=90^{\circ}\right)=8.49 \cdot 10^{-5} \%$. With the fibre composite transmission model from Eq. 2 in Sect. 2.3 we can determine the efficiency parameter $Q_{e x t}$ for both given fibre orientations. For this calculation we applied the material parameters given in Sect. 3. Thus, for $\gamma=0^{\circ}$ this leads to $Q_{\text {ext }, 0} \approx 0.5565$ and for $\gamma=90^{\circ}$ to $Q_{\text {ext }, 90} \approx 0.5560$. Below, we examine the effect of intra material beam propagation on the size of the reflected and imaged laser spot.

\section{Discussion}

This section discusses the major findings of this study and links them to the related research. Goniometer measurements provide necessary data for the evaluation of the direction dependent reflection properties of CFRP. These information are used for the investigations in this paper. Thus this is in accordance with the conclusions from both publications from Li et al. [32, 54], Rabal et al. [33] as well as Martinez and Hartmann [34]. In particular, the angle-restricted BRDF modelling applied in this paper is very well suited to determine the required reflection characteristics for this application. Furthermore, the Fresnel equations provide a convenient way to approximate the refraction index of a composite 
through fitting the peaks of its specular reflectance, as already stated from Stokes-Griffin and Compston [16]. Snell's law also gives reasonably accurate approximations for the critical angle for total internal reflection despite looking at a strongly non-isotropic material.

In order to answer the introduced research question we found, that a very astonishing result from the measurement of the Hexcel HexPly 8552 IM7 optical properties is the strongly deviating reflection behaviour between a $\gamma=0^{\circ}$ and $\gamma=90^{\circ}$ rotated material sample. The FWHM is up to eight times larger for $\gamma=90^{\circ}$ than for $\gamma=0^{\circ}$. In contrast, the maximum degree of reflection at $\gamma=90^{\circ}$ is up to $40 \%$ lower than for $\gamma=0^{\circ}$. These results align rather well with the findings from Grouve [38] as well as StokesGriffin and Compston [16], which model the reflectance behaviour of several individual fibres with a single large cylinder. With respect to the LLSS inspection application in composite manufacturing, these findings are especially relevant for lenses with a wide viewing angle. They capture a significant gradient in the degree of reflection along the width of the material. Furthermore, these findings are essential for scanning transverse to the fibre orientation. In addition, it should be noted that the largest degree of reflection arises for equal irradiation and detection angles.

In future research, we need to investigate the change of the optical properties of the prepreg material under different process temperatures. Furthermore, the behaviour of the subsequent evaluation algorithms in the acutal inspection system is of interest for different optical material characteristics.

Below the results of this study are summarised and the added value for the community is highlighted.

\section{Conclusion}

In this section we summarise the key findings of this paper and highlight the contribution to industry and research. Accordingly, the reflection and scattering properties of the Hexcel HexPly 8552 IM7 test material are investigated using a Goniometer. In these investigations, the strongly varying reflection qualities of the fibre material for $\gamma=90^{\circ}$ fibre orientation are noticeable. Compared to $\gamma=0^{\circ}$, the full width at half maximum can be eight times larger for $\gamma=90^{\circ}$. Whereas, the maximum degree of reflection at $\gamma=90^{\circ}$ is only $60 \%$ of the $\gamma=0^{\circ}$ maximum.

The outcome of this study is rather beneficial for developers and users of optical inspection systems for the visual part testing. Our results assist the material specific choice of lenses and the optical design of the device.

In future research the optical material properties for various process temperatures as well as the resulting behaviour of the subsequent image processing algorithms need to be evaluated.

Funding Open Access funding enabled and organized by Projekt DEAL. This research is part of the project DHiiP-AIR and was financially supported by the Federal Ministry for Economic Affairs and Energy. This project has received funding from the Federal Ministry for Economic Affairs and Energy under the funding code No. 20W1911F. 


\title{
Supported by:
}

\author{
侄 Federal Ministry \\ for Economic Affairs \\ and Energy

\section{on the basis of a decision \\ by the German Bundestag}

Availability of Data and Material The corresponding image data is made available on request.

Code Availability The corresponding Python code is made available on request.

\section{Declarations}

Conflicts of Interest The authors declare that they have no conflict of interest.

Open Access This article is licensed under a Creative Commons Attribution 4.0 International License, which permits use, sharing, adaptation, distribution and reproduction in any medium or format, as long as you give appropriate credit to the original author(s) and the source, provide a link to the Creative Commons licence, and indicate if changes were made. The images or other third party material in this article are included in the article's Creative Commons licence, unless indicated otherwise in a credit line to the material. If material is not included in the article's Creative Commons licence and your intended use is not permitted by statutory regulation or exceeds the permitted use, you will need to obtain permission directly from the copyright holder. To view a copy of this licence, visit http://creativecommons.org/licenses/by/4.0/.

\section{References}

1. Marsh, G.: Airbus A350 XWB update. Reinf. Plast. 54(6), 20-24 (2010). https://doi.org/10.1016/ s0034-3617(10)70212-5

2. Mcllhagger, A., Archer, E., McIlhagger, R.: Manufacturing processes for composite materials and components for aerospace applications. In: Irving, P., Soutis, C. (eds.) Polymer Composites in the Aerospace Industry, pp. 59-81. Elsevier (2020). https://doi.org/10.1016/b978-0-08-102679-3.00003-4

3. Cemenska, J., Rudberg, T., Henscheid, M.: Automated in-process inspection system for AFP machines. SAE Int. J. Aerosp. 8(2), 303-309 (2015). https://doi.org/10.4271/2015-01-2608

4. Weimer, C., Friedberger, A., Helwig, A., Heckner, S., Buchmann, C., Engel, F.: Increasing the productivity of CFRP production processes by robustness and reliability enhancement. In: CAMX 2016 - The Composites and Advanced Materials Expo and Conference. Airbus Group Innovations, 81663 Munich, Germany; AirbusInfactory Solutions GmbH, 81663 Munich, Germany (2016). https://www.researchgate.net/ profile/Christian_Weimer/publication/308778487_INCREASING_THE_PRODUCTIVITY_OF_CFRP_ PRODUCTION_PROCESSES_BY_ROBUSTNESS_AND_RELIABILITY_ENHANCEMENT/links/ 57efa78208ae886b8975147a.pdf

5. Black, S.: Improving composites processing with automated inspection. compositesworld (2018). https://www.compositesworld.com/articles/improving-composites-processing-with-automatedinspection

6. Rudberg, T., Nielson, J., Henscheid, M., Cemenska, J.: Improving AFP cell performance. SAE Int. J. Aerosp. 7(2), 317-321 (2014). https://doi.org/10.4271/2014-01-2272 
7. Rudberg, T.: Webinar: Building AFP system to yield extreme availability. CompositesWorld (2019). Video

8. Eitzinger, C.: Inline inspection helps accelerate production by up to $50 \%$. Lightweight Design worldwide (2019)

9. Meister, S., Möller, N., Stüve, J., Groves, R.M.: Synthetic image data augmentation for fibre layup inspection processes: Techniques to enhance the data set. J. Intell. Manuf. (2021). https://doi.org/10. 1007/s10845-021-01738-7

10. Ucan, H., Scheller, S., Nguyen, D.C., Nieberl, D., Beumler, T., Haschenburger, A., Meister, S., Kappel, E., Prussak, R., Deden, D., Mayer, M., Pantelelis, N., Zapp, P., Hauschild, B., Menke, N.: Automated, quality assured and high volume oriented production of fiber metal laminates (FML) for the next generation of passenger aircraft fuselage shells. In: The Fourth International Symposium on Automated Composites Manufacturing (2019). https://elib.dlr.de/127353/

11. Meister, S., Wermes, M.A.M., Stueve, J., Groves, R.M.: Algorithm assessment for layup defect segmentation from laser line scan sensor based image data. In: Zonta, D., Huang, H. (eds.) Sensors and Smart Structures Technologies for Civil, Mechanical, and Aerospace Systems 2020. SPIE (2020). https://doi.org/10.1117/12.2558434

12. Louët, V.L., Rousseau, B., Corre, S.L., Boyard, N., Tardif, X., Delmas, J., Delaunay, D.: Directional spectral reflectivity measurements of a carbon fibre reinforced composite up to $450{ }^{\circ} \mathrm{C}$. Int. J. Heat Mass Transf. 112, 882-890 (2017). https://doi.org/10.1016/j.ijheatmasstransfer.2017.04.125

13. Adibekyan, A., Kononogova, E., Monte, C., Hollandt, J.: Review of PTB measurements on emissivity, reflectivity and transmissivity of semitransparent fiber-reinforced plastic composites. Int. J. Thermophys. 40(4), (2019). https://doi.org/10.1007/s10765-019-2498-0

14. Francesco, M.D., Veldenz, L., Dell'Anno, G., Potter, K.: Heater power control for multi-material, variable speed automated fibre placement. Compos. A: Appl. Sci. Manuf. 101, 408-421 (2017). https://doi.org/10.1016/j.compositesa.2017.06.015

15. Danezis, A., Williams, D., Edwards, M., Skordos, A.: Heat transfer modelling of flashlamp heating for automated tape placement of thermoplastic composites. Compos. A: Appl. Sci. Manuf. 106381 (2021). https://doi.org/10.1016/j.compositesa.2021.106381

16. Stokes-Griffin, C., Compston, P.: Optical characterisation and modelling for oblique near-infrared laser heating of carbon fibre reinforced thermoplastic composites. Opt. Lasers Eng. 72, 1-11 (2015). https://doi.org/10.1016/j.optlaseng.2015.03.016

17. Meister, S., Grundhöfer, L., Stüve, J., Groves, R.M.: Imaging sensor data modelling and evaluation based on optical composite characteristics. Int. J. Adv. Manuf. Technol. (2021). https://doi.org/10. 1007/s00170-021-07591-5

18. Parmar, H., Khan, T., Tucci, F., Umer, R., Carlone, P.: Advanced robotics and additive manufacturing of composites: towards a new era in industry 4.0. Mater. Manuf. Process. 1-35 (2021). https:// doi.org/10.1080/10426914.2020.1866195

19. Maass, D.: Automated dry fiber placement for aerospace composites. In: Composites Manufacturing 2012. Danobat (2012)

20. Campbell, F.: Manufacturing Processes for Advanced Composites. Elsevier Science \& Technology (2004). https://www.ebook.de/de/product/6827737/manufacturing_processes_for_advanced_ composites.html

21. Oromiehie, E., Prusty, B.G., Compston, P., Rajan, G.: Automated fibre placement based composite structures: Review on the defects, impacts and inspections techniques. Compos. Struct. 224, 110987 (2019)

22. Lengsfeld, H., Fabris, F.W., Krämer, J., Lacalle, J., Altstädt, V.: Faserverbundwerkstoffe. Hanser Fachbuchverlag (2014). https://www.ebook.de/de/product/22746074/hauke_lengsfeld_felipe_wolff_ fabris_johannes_kraemer_javier_lacalle_volker_altstaedt_faserverbundwerkstoffe.html

23. Atkinson, G.A., Thornton, T.J., Peynado, D.I., Ernst, J.D.: High-precision polarization measurements and analysis for machine vision applications. In: 2018 7th European Workshop on Visual Information Processing (EUVIP). IEEE (2018). https://doi.org/10.1109/euvip.2018.8611762

24. Schöberl, M., Kasnakli, K., Nowak, A.: Measuring strand orientation in carbon fiber reinforced plastics (CFRP) with polarization. In: 19th World Conference on Non-Destructive Testing 2016. (2016)

25. Denkena, B., Schmidt, C., Völtzer, K., Hocke, T.: Thermographic online monitoring system for automated fiber placement processes. Compos. Part B Eng. 97, 239-243 (2016). https://doi.org/10. 1016/j.compositesb.2016.04.076

26. Gregory, E.D., Juarez, P.D.: In-situ thermography of automated fiber placement parts. In: AIP Conference Proceedings (2018). https://doi.org/10.1063/1.5031551 
27. Schmidt, C., Hocke, T., Denkena, B.: Artificial intelligence for non-destructive testing of CFRP prepreg materials. Prod. Eng. (2019). https://doi.org/10.1007/s11740-019-00913-3

28. Gardiner, G.: Zero-defect manufacturing of composite parts. CompositesWorld (2018). https://www. compositesworld.com/blog/post/zero-defect-manufacturing-of-composite-parts. Accessed: 2019-06-18

29. Black, S.: Improving composites processing with automated inspection, part II. compositesworld (2018). https://www.compositesworld.com/articles/improving-composites-processing-with-automatedinspection-part-ii. Accessed: 2019-06-19

30. Sun, S., Han, Z., Fu, H., Jin, H., Dhupia, J.S., Wang, Y.: Defect characteristics and online detection techniques during manufacturing of FRPs using automated fiber placement: A review. Polymers 12(6), 1337 (2020). https://doi.org/10.3390/polym12061337

31. Schmitt, R., Orth, A., Niggemann, C.: A method for edge detection of textile preforms using a lightsection sensor for the automated manufacturing of fibre-reinforced plastics. In: Osten, W., Gorecki, C., Novak, E.L.(eds.) Optical Measurement Systems for Industrial Inspection V. SPIE (2007). https://doi. org/10.1117/12.726177

32. Li, H., Chen, M., Deng, C., Liao, N., Rao, Z.: Versatile four-axis gonioreflectometer for bidirectional reflectance distribution function measurements on anisotropic material surfaces. Opt. Eng. 58(12), 1 (2019). https://doi.org/10.1117/1.oe.58.12.124106

33. Rabal, A.M., Ferrero, A., Campos, J., Fontecha, J.L., Pons, A., Rubiño, A.M., Corróns, A.: Automatic gonio-spectrophotometer for the absolute measurement of the spectral BRDF at in- out-of-plane and retroreflection geometries. Metrologia 49(3), 213-223 (2012). https://doi.org/10.1088/0026-1394/49/3/ 213.

34. Martinez, M.L., Hartmann, T.: Multispectral gonioreflectometer facility for directional reflectance measurements and its use on materials and paints. In: Stein, K.U., Schleijpen R. (eds.) Target and Background Signatures IV. SPIE (2018). https://doi.org/10.1117/12.2325711

35. Forest, J., Salvi, J., Cabruja, E., Pous, C.: Laser stripe peak detector for $3 \mathrm{~d}$ scanners. a FIR filter approach. In: Proceedings of the 17th International Conference on Pattern Recognition, 2004. ICPR 2004. IEEE (2004). https://doi.org/10.1109/icpr.2004.1334612

36. Miesen, N., Sinke, J., Groves, R.M., Benedictus, R.: Simulation and detection of flaws in pre-cured CFRP using laser displacement sensing. Int. J. Adv. Manuf. Technol. 82(1-4), 341-349 (2015). https:// doi.org/10.1007/s00170-015-7305-X

37. Xu, H., Hu, J., Yu, Z.: Absorption behavior analysis of carbon fiber reinforced polymer in laser processing. Opt. Mater. Express 5(10), 2330 (2015). https://doi.org/10.1364/ome.5.002330

38. Grouve, W.: Weld strength of laser-assisted tape-placed thermoplastic composites. phdthesis, University of Twente (2012). https://doi.org/10.3990/1.9789036533928

39. Riviere, N., Ceolato, R., Hespel, L.: Multispectral polarized brdf: design of a highly resolved reflectometer and development of a data inversion method. Opt. Appl. 42(1), 7-22 (2012). https://doi.org/10. 5277/oa120101

40. Nicodemus, F.E.: Directional reflectance and emissivity of an opaque surface. Appl. Opt. 4(7), 767 (1965). https://doi.org/10.1364/ao.4.000767

41. Stover, J.: Optical scattering : measurement and analysis, 3 edn. Spie Press, Place of publication not identified (2016). https://spie.org/Publications/Book/975276?SSO=1

42. Dingemans, L.M., Papadakis, V.M., Liu, P., Adam, A.J.L., Groves, R.M.: Quantitative coating thickness determination using a coefficient-independent hyperspectral scattering model. Journal of the European Optical Society-Rapid Publications 13(1) (2017). https://doi.org/10.1186/s41476-017-0068-2

43. Beer, A.: Bestimmung der absorption des rothen lichts in farbigen flüssigkeiten. Annalen der Physik und Chemie 162(5), 78-88 (1852). https://doi.org/10.1002/andp.18521620505. https://onlinelibrary. wiley.com/doi/abs/10.1002/andp.18521620505

44. Kubelka, P., Munk, F.: Ein beitrag zur optik der farbanstriche. Z. Tech. Phys. 12, 593-601 (1931)

45. Hohmann, A., ElMaklizi, A., Foschum, F., Voit, F., Bergmann, F., Simon, E., Reitzle, D., Kienle, A.: Optics of carbon fiber-reinforced plastics - a theoretical and an experimental study. J. Quant. Spectrosc. Radiat. Transf. 180, 70-76 (2016). https://doi.org/10.1016/j.jqsrt.2016.04.015

46. Zhang, P., Cao, P., Yang, Y., Guo, P., Chen, S., Zhang, D.: Simulation of a machine vision system for reflective surface defect inspection based on ray tracing. Appl. Opt. 59(8), 2656 (2020). https://doi.org/ 10.1364/ao.385486

47. Iba, H., Kagawa, Y.: Light transmittance of continuous fibre-reinforced composites: Analysis, model experiment and parametric study. Philos. Mag. B 78(1), 37-52 (1998). https://doi.org/10.1080/ 13642819808206725

48. Iba, H., Naganuma, T., Matsumura, K., Kagawa, Y.: Fabrication of transparent continuous oxynitride glass fiber-reinforced glass matrix composite. J. Mater. Sci. 34(23), 5701-5705 (1999). https://doi.org/ 10.1023/a:1004741714469 
49. Pascual, C., de Castro, J., Schueler, A., Vassilopoulos, A.P., Keller, T.: Total light transmittance of glass fiber-reinforced polymer laminates for multifunctional load-bearing structures. J. Compos. Mater. 48(29), 3591-3604 (2013). https://doi.org/10.1177/0021998313511653

50. Kasap, S., Capper, P.: Springer Handbook of Electronic and Photonic Materials, 2 edn. Springer International Publishing (2017). https://doi.org/10.1007/978-3-319-48933-9

51. Hexcel Corporation: Hexply 8552 - datasheet. techreport, Hexcel Corporation (2020). https://www. hexcel.com/user_area/content_media/raw/HexPly_8552_eu_DataSheet.pdf

52. Hexcel Corporation: Hextow im 7 - carbon fiber. techreport, Hexcel Corporation (2020). https://www. hexcel.com/user_area/content_media/raw/IM7_HexTow_DataSheet.pdf

53. Opsira GmbH: gonio’2pi. techreport, Opsira GmbH, Leibnizstraße 20, 88250 Weingarten, Germany (2019). https://www.opsira.de/fileadmin/benutzerdaten/opsira-de/pdf/Product_information_near-field_ goniophotometer_gonio_2pi_-_E_-_V00263114_-_opsira.pdf

54. Li, H., Foo, S.C., Torrance, K.E., Westin, S.H.: Automated three-axis gonioreflectometer for computer graphics applications. Opt. Eng. 45(4), 1 - 11 (2006). https://doi.org/10.1117/1.2192787

Publisher's Note Springer Nature remains neutral with regard to jurisdictional claims in published maps and institutional affiliations. 